\title{
Genetic determinants of bone mineral content in premature infants
}

\author{
M C Backström, A Mahonen, M Ala-Houhala, H Sievänen, P Mäenpää, A M Koivisto,
} A Olkku, R Mäki, M Mäki

\begin{abstract}
Genetic determinants of bone mineral content in prematurely born children at 3 months and 9-11 years of age were studied. The findings suggest that multiple genes are involved in the regulation of site specific bone mass accumulation during childhood.
\end{abstract}

(Arch Dis Child Fetal Neonatal Ed 2001;85:F214-F216)

Keywords: bone mineral status; collagen; insulin-like growth factor I; oestrogen; vitamin D receptor

Peak bone mass in early adulthood is an important determinant of risk of fracture through the rest of life. ${ }^{1}$ It is influenced by environmental factors such as calcium intake and exercise, but genetics is considered to play a major role. ${ }^{2}$ In preterm infants, the environmental influence on bone mineral content (BMC) is strong and well established. ${ }^{3}$ This study is the first attempt to shed light on the genetic effect on bone mineral accretion in children born preterm.

\section{Methods}

Thirty nine children born before the gestational age of 37 weeks were enlisted in a study to assess the effect of early diet on BMC. ${ }^{4}$ Except for minor illnesses, the children were free from any chronic diseases. BMC of the left forearm, specifically at a site one third of the ulnar length measured proximally from the distal ulnar styloid (one third site), was measured by single photon absorptiometry ${ }^{5}$ at 3 months of age in 16 of these infants (mean (SD) gestational age 29.6 (3.0) weeks and birth weight 1263 (415) g). At 9-11 years of age, BMC was measured in all children by dual energy $x$ ray absorptiometry (Norland XR-26, Norland Corp, Fort Atkinson, Wisconsin, USA) ${ }^{6}$ at the lumbar spine (L2-L4) and distal left forearm in addition to the one third site. The mean (SD) gestational age of the whole group was 31.3 (3.2) weeks and the mean (SD) birth weight was 1483 (442) g.

Genomic DNA was extracted and purified from EDTA blood samples using a QIAamp Blood Kit (Qiagen GmbH, Hilden, Germany). For restriction fragment length polymorphism studies of vitamin D receptor (VDR) and oestrogen receptor $(\mathrm{ER} \alpha)$ genes carrying the polymorphic restriction sites, genomic DNA was amplified by polymerase chain reaction (PCR) using gene specific primers. ${ }^{78}$ After amplification, the PCR products were digested with restriction endonucleases ( $A p a \mathrm{I}, T a q \mathrm{I}$, and FokI for VDR; PvuII for ER $\alpha$ ), and the resulting fragments were separated in $2 \%$ agarose gel. The alleles were defined by capital letters in the absence of a restriction site and small letters in the presence of a given restriction site. The 569 bp fragment of genomic DNA containing the polymorphic portion (a $G$ to $T$ substitution) of the $\mathrm{Sp} 1$ binding site in intron 1 of the collagen type I $\alpha 1$ (COLIA1) gene was amplified by PCR under similar reaction conditions to those described above. The upstream primer was 5'-GGAAAGTAAAGCCAGGGATG-3' and the downstream primer was 5'AACTCCAACCTCAGCCCATT-3'. After amplification, the PCR product was digested with PflMI endonuclease producing $379 \mathrm{bp}$ and $190 \mathrm{bp}$ fragments. The PflMI recognises the sequence CCANNN/NTGG where the last $\mathrm{G}$ represents the polymorphic site of the Sp1 binding region. ${ }^{9}$ According to Grant and coworkers, ${ }^{10}$ the allele $S$ was designated as absence of the $\mathrm{G}$ to $\mathrm{T}$ transition (the presence of a restriction site in this study).

The polymorphic dinucleotide repeat upstream of the transcription start site of the insulin-like growth factor (IGF)-I gene was also amplified using gene specific primers. ${ }^{11}$ One primer was fluorescently labelled (Cy5; Amersham Pharmacia Biotech, Little Chalfont, Bucks, UK). The variation in length of labelled PCR products was analysed using an ALFexpress DNA sequencer and ALFwin Fragment Analyser software (Amersham Pharmacia Biotech AB, Uppsala, Sweden).

Between group differences were tested by analysis of covariance. The genotype was used as a factor and the weight at the time of the BMC measurement was used as a covariate. For the analysis of the effect of the IGF-I genotype, all different base pair lengths were separately analysed. The children were classified into three groups: (a) homozygotes for a certain length of DNA; $(b)$ heterozygotes for that length of DNA; (c) those who did not have that length of DNA. Analysis of variance was performed with these groups for all basepair lengths. When the effect of different genotypes on the change in BMC was being evaluated, the difference between the BMC at 3 months and 9-11 years of age was used as a factor and the change in body weight during the same time period was used as a covariate. Because of the multiple comparisons, the $\alpha$ level was set at 0.01 . The prevalences of VDR genotypes were compared by cross tabulation and $\chi^{2}$ test.

The study was approved by the ethics committee of Tampere University Hospital, and written informed consent was obtained from the parents. 


\section{Results}

At 3 months of age, children with the VDR polymorphism TaqI genotype tt tended to have higher BMC than those with the genotype $\mathrm{Tt}$ or TT (table 1). The VDR ApaI genotype had no impact on BMC at 3 months of age. The distributions of the VDR FokI and ER $\alpha$ PvuII and COLIA1 genotypes were not representative at 3 months of age and no conclusions could be drawn from these genotypes.

As to IGF-I polymorphism, the size of the PCR product varied from 182 to $196 \mathrm{bp}$, a length of $190 \mathrm{bp}$ being the most common in our study population. This genetic determinant had no influence on BMC at 3 months of age (data not shown)

At 9-11 years of age, the TaqI and ApaI genotypes were associated with each other $(\mathrm{p}<0.01)$, whereas the FokI genotype was not associated with TaqI or ApaI ( $\mathrm{p}=0.38$ and $\mathrm{p}=0.45)$. The VDR genotype FokI was the only genetic determinant significantly $(\mathrm{p}<0.01)$ associated with BMC at the distal forearm (table 1).

The $190 \mathrm{bp}$ allele of the IGF-I gene tended to have an effect on the increase in forearm shaft BMC from 3 months to 9-11 years of age $(p=0.02)$. The children who were homozygotes or heterozygotes for the $190 \mathrm{bp}$ allele of the IGF-I allele showed a slower increase in forearm shaft BMC from 3 months to 9-11 years of age than those without this allele ( 374 $\mathrm{g} / \mathrm{cm} v 493 \mathrm{~g} / \mathrm{cm}, \mathrm{p}<0.01)$. None of the other genotypes studied were related to the change in BMC of the forearm shaft from 3 months to 9-11 years of age.

\section{Discussion}

Most studies of genetic influence on BMC have been performed on perimenopausal and postmenopausal women. Some studies have been carried out on young adults and children, but to our knowledge this is the first study to consider the effect of genetics on BMC in prematurely born children. We found a tendency towards higher BMC at 3 months of age in the children with the VDR TaqI tt genotype compared with those with the Tt or TT genotype (table 1). The TaqI polymorphism was not related to birth weight or weight at 3 months of age. In contrast, in infants born at term, the VDR genotype tt has been shown to be associated with higher body weight adjusted total $\mathrm{BMC}$ at 1 year of age. ${ }^{12}$ This genotype is also related to a higher body weight in 1 year old term infants, but both of these associations disappear later in childhood. ${ }^{13}$ The present insignificant association of VDR TaqI polymorphism with BMC in early life may be related to the small sample size, but one cannot exclude the possibility that the strong environmental factors ${ }^{3}$ present during the neonatal period of preterm infants may well conceal a potential genetic effect.

At 9-11 years of age, only the polymorphism at the FokI site of the VDR gene was associated with bone mineral status (table 1). The VDR ApaI and TaqI genotypes were closely related to each other. $A p a \mathrm{I}$ and $T a q \mathrm{I}$ enzymes identify polymorphisms at the 3' end of the VDR gene and do not alter the coding sequence of this gene, in contrast with FokI which identifies the polymorphism at the start codon of the VDR gene. ${ }^{8}$ If the FokI restriction site is present (presence denoted by $\mathrm{f}$ ), the VDR protein is three amino acids longer than if the restriction site is absent (absence denoted by F). This may result in a greater effect of FokI polymorphism than $A p a \mathrm{I}$ and TaqI polymorphism on VDR function and thus BMC. The longer VDR protein has been speculated to result in mild calcitriol resistance. ${ }^{8}$ In accord with this, the children in the present study with the genotype ff had lower body weight adjusted BMC at the

Table 1 Bone mineral content (BMC) of the radial shaft, forearm shaft, and distal forearm in prematurely born children with different vitamin $D$ receptor (VDR), oestrogen receptor (ERa) and collagen type I a 1 (COLIA1) genotypes

\begin{tabular}{|c|c|c|c|c|c|c|c|c|c|c|}
\hline & \multicolumn{3}{|c|}{ At 3 months } & \multicolumn{7}{|c|}{ At 9 to 11 years } \\
\hline & \multicolumn{3}{|c|}{ Radial shaft $B M C(\mathrm{~g} / \mathrm{cm})$} & \multicolumn{3}{|c|}{ Forearm shaft $B M C(g)$} & \multicolumn{2}{|c|}{ Distal forearm $B M C(g)$} & \multicolumn{2}{|c|}{ Lumbar BMC (g) } \\
\hline & $n$ & Mean (SD) & p Value & $n$ & Mean (SD) & p Value & Mean (SD) & $p$ Value & Mean (SD) & $p$ Value \\
\hline \multicolumn{11}{|l|}{ VDR } \\
\hline \multicolumn{11}{|l|}{ ApaI } \\
\hline aa & 4 & $0.09(0.03)$ & \multirow{3}{*}{0.46} & 8 & $0.80(0.19)$ & \multirow{3}{*}{0.88} & $0.86(0.20)$ & \multirow{3}{*}{0.87} & $19.53(5.57)$ & \multirow{3}{*}{0.39} \\
\hline $\mathrm{Aa}$ & 7 & $0.11(0.04)$ & & 19 & $0.80(0.17)$ & & $0.90(0.25)$ & & $19.60(4.58)$ & \\
\hline $\mathrm{AA}$ & 5 & $0.09(0.02)$ & & 12 & $0.79(0.21)$ & & $0.91(0.30)$ & & $20.95(4.93)$ & \\
\hline \multicolumn{11}{|l|}{ FokI } \\
\hline ff & 2 & $0.09(0.05)$ & & 5 & $0.86(0.23)$ & \multirow{3}{*}{0.12} & $0.93(0.32)$ & \multirow{3}{*}{$<0.01$} & $22.19(6.99)$ & \multirow{3}{*}{0.74} \\
\hline $\mathrm{Ff}$ & 10 & $0.10(0.04)$ & & 21 & $0.72(0.17)$ & & $0.82(0.25)$ & & $18.49(4.63)$ & \\
\hline FF & 4 & $0.08(0.02)$ & & 13 & $0.88(0.13)$ & & $1.00(0.21)$ & & $21.60(3.53)$ & \\
\hline \multicolumn{11}{|l|}{ TaqI } \\
\hline $\mathrm{tt}$ & 2 & $0.11(0.01)$ & \multirow{3}{*}{0.02} & 6 & $0.76(0.26)$ & \multirow{3}{*}{0.53} & $0.94(0.40)$ & \multirow{3}{*}{0.43} & $21.01(6.39)$ & \multirow{3}{*}{0.34} \\
\hline $\mathrm{Tt}$ & 6 & $0.09(0.04)$ & & 20 & $0.80(0.16)$ & & $0.91(0.25)$ & & $20.44(4.60)$ & \\
\hline $\mathrm{TT}$ & 8 & $0.09(0.03)$ & & 13 & $0.80(0.19)$ & & $0.85(0.18)$ & & $18.86(4.51)$ & \\
\hline \multicolumn{11}{|l|}{$\mathrm{ER} \alpha$} \\
\hline \multicolumn{11}{|l|}{ Рvи II } \\
\hline pp & 1 & 0.12 & & 8 & $0.81(0.24)$ & \multirow{3}{*}{0.65} & $0.90(0.36)$ & \multirow{3}{*}{0.36} & $21.29(7.37)$ & \multirow{3}{*}{0.87} \\
\hline $\mathrm{pP}$ & 12 & $0.09(0.04)$ & & 23 & $0.81(0.17)$ & & $0.91(0.25)$ & & $20.00(4.20)$ & \\
\hline PP & 3 & $0.09(0.01)$ & & 8 & $0.72(0.13)$ & & $0.83(0.14)$ & & $18.74(2.99)$ & \\
\hline \multicolumn{11}{|l|}{ COLIA1 } \\
\hline ss & 1 & 0.16 & & 1 & 0.98 & & 0.91 & & 19.08 & \\
\hline Ss & 1 & 0.10 & & 6 & $0.86(0.18)$ & & $1.07(0.36)$ & & $21.70(5.61)$ & \\
\hline SS & 14 & 0.09 & & 31 & $0.78(0.18)$ & & $0.86(0.23)$ & & $19.63(4.81)$ & \\
\hline
\end{tabular}

$\mathrm{p}$ Values were obtained by analysis of covariance with genotypes aa, Aa, and AA, ff, Ff, and FF, tt, Tt, and TT, pp, pP, and PP, or ss, Ss, and SS as factors and weight at time of measurement of bone mineral status as covariate.

The $\mathrm{p}$ values for the VDR FokI, ER $\alpha$ PvuII and COLIA1 genotypes are not reported because the distributions are not representative. 
distal radius at 9-11 years of age than the children with the genotype FF. In contrast, the Ff heterozygotes in our study had the lowest BMC at the distal radius, a finding that we cannot explain. The fact that no effect of FokI polymorphism was seen at the lumbar site is in agreement with the suggestion that the genetic influence is not uniform over the skeleton. ${ }^{14}$

Rosen and coworkers ${ }^{11}$ found that the IGF-I 192/192 homozygotes had lower levels of serum IGF-I than the other genotypes. Low serum levels of IGF-I have been shown to be associated with low BMC. ${ }^{15}$ In the present study, only one child was found to be a 192/192 homozygote, thus the effect of this genotype was impossible to evaluate. The children who were homozygotes or heterozygotes for the 190 bp allele of IGF-I showed a significantly slower increase in forearm shaft BMC from 3 months to 9-11 years age compared with those without this allele.

In conclusion, multiple genes may be involved in the regulation of site specific accumulation of bone mass during childhood. However, it must be kept in mind that the small sample size of this study limits its statistical power to detect true associations, and a type II error cannot be excluded.

1 Johnston C C, Slemenda CW. Peak bone mass, bone loss and risk of fracture. Osteoporos Int 1994;4(suppl 1):S43-5.

2 Pocock N A, Eisman J A, Hopper J L, et al. Genetic determinants of bone mass in adult. $\mathcal{F}$ Clin Invest 1987;80:706-10
3 Backström M C, Kuusela A-L, Mäki R. Metabolic bone disease of prematurity. Ann Med 1996;28:275-82.

4 Backström M C, Mäki R, Kuusela A-L, et al. The long-term effect of early mineral, vitamin $\mathrm{D}$ and breast milk intake on bone mineral status in 9-11 years old children born prematurely. F Pediatr Gastroenterol Nutr 1999;29:575-82.

5 Karjalainen P. A method for determination of the mineral content and the mineral density in the distal radius using gamma ray attenuation. Ann Clin Res 1973;5:231-7.

6 Sievänen H, Kannus P, Nieminen V, et al. Estimation of various mechanical characteristics of human bones using dual energy X-ray absorptiometry: methodology and precision. Bone 1996;18:17S-28S.

7 Castagnoli A, Mastri I, Bernardi F, et al. PvuII RFLP inside the human estrogen receptor gene. Nucleic Acids Res 1987;15:866.

8 Gross C, Eccleshall T R, Malloy P J, et al. The presence of polymorphism at the translation initiation site of vitamin $\mathrm{D}$ receptor gene is associated with low bone mineral density in postmenopausal Mexican-American Women. $f$ Bone Miner Res 1996;11:1850-5.

9 Vinkanharju A, Risteli J, Risteli L. New method for detecting the Sp1 polymorphism in the COLIA1 gene. Bone 1998;23:S348.

10 Grant S F, Reid D M, Blake G, et al. Reduced bone density and osteoporosis associated with a polymorphic Sp1 binding site in the collagen type I alpha 1 gene. Nat Genet 1996;14:203-5.

11 Rosen CJ, Kurland ES, Vereault D, et al. Association between serum insulin growth factor-I (IGF-I) and a simple sequence repeat in IGF-I gene: implications for genetic ple sequence repeat in IGF-I gene: implications for genetic 1998;83:2286-90.

12 Specker BL, Williams LA, Kalkwarf H, et al. Vitamin D receptor genotype and its relation to bone growth and bone mineralisation in infants 3 to 12 months of age. $\mathcal{f}$ Bone Miner Res 1995;10:S188.

13 Chang T, Yu T, Garnett S, et al. Vitamin D receptor alleles predict growth and bone density in girls. Arch Dis Child 1998;79:488-93.

14 Rubin LA, Hawker GA, Peltekova VD, et al. Determinants of peak bone mass: clinical and genetic analyses in young
female Canadian cohort. $\mathcal{F}$ Bone Miner Res 1999;14:63343.

15 Moreira-Andres MN, Papapietro K, Canizo FJ, et al. Correations between bone mineral density, insulin-like growth factor I and auxological variables. Eur $f$ Endocrinol 1995;132:573-9. 